manuscripta math. 10, 297 - 306 (1973)

(C) by Springer-Verlag 1973

\title{
A CONTRIBUTION TO SEGAL ALGEBRAS
}

\author{
Michael Leinert
}

Let $A$ and $B$ be Banach algebras. If $B$ is an abstract Segal algebra in $A$, we have a bijective correspondence between the strictly irreducible representations of $A$ and those of $B$. This gives a bijective correspondence for maximal modular left ideals. If $A$ and $B$ have approximate right units, we obtain a bijective correspondence for right resp. two-sided ideals. For two-sided ideals this correspondence preserves the property of an ideal having approximate right units. This generalizes a Theorem by H. Reiter.

Notation. For a subset 1 of a topological space $X$ we denote by $T^{X}$ the closure of $I$ in X. If $B$ is a Banach algebra, Rad B is its radical and $B^{\prime}$ is the space of all continuous linear functionals on $B$. Two subsets $D \subset B$ and $E<B^{\prime}$ are called orthogonal $(D \perp E)$, if $d(e)=0$ for all $d \in D$, e $\in E$. Throughout this note $A$ and $B$ will denote Banach algebras. I should Iike to thank H. Behncke for drawing my attention to the paper [1] by B. A. Barnes.

\section{Preliminaries}

Definition. Let $A$ be a Banach algebra with norm I I A and let $B$ be a dense left ideal in $A$ such that

(1.1) B is a Banach algebra with respect to some norm I I $B$ *)

(1.2) There is a constant $K>0$ such that

$$
|b|_{A} \leq K|b|_{B}
$$

for $a l l b \in B$.

Then B is called an abstract Segal algebra in A.

*) It would be sufficient to require $B$ to be a Banach space. 
In case that $A$ is semi-simple, condition (1.2) in the above definition may be omitted since it is fulfilled automatically. This is proved as in $[1]$, Proposition 2.2, using the following modification of $[1]$, Proposition 1.1:

Lemma (1.3). Let $B$ be a dense left ideal in $A$ and let $M$ be a maximal modular left ideal in A. Then

(i) B acts strictly irreducibly on $A / M$

( $i$ i) $B \cap M$ is a maximal modular left ideal in B.

Proof: (i) Since the left regular representation $\pi$ of $A$ on the space $A / M$ is continuous and $B$ is dense in $A$, we have for $\xi \in A / M, \xi \neq 0$

$$
\overline{\pi(B)}^{A / M}=\pi(A) \xi=A / M
$$

This implies $\pi(B) \xi \neq 0$. Since $\pi(B) \xi$ is $\pi(A)$ - invariant, we have $\pi(B) \xi=A / M$.

(ii) Let $\xi=u+M$, where $u$ is a right identity for $A$ modulo $M$. Then $M$ is the annihilator of $\xi$ in $A$, and $B \cap M$ is the annihilator of $\xi$ in $B$, which is a maximal modular left ideal of B by (i).

The proof given by B. A. Barnes ( [1], Proposition 1.1) for a twosided ideal $B$ is a purely algebraic one, however it seems that in the case of a left ideal one has to use topology for (i).

If $B$ is a left ideal in $A$ and condition (1.2) holds, in particular if $B$ is an abstract Segal algebra in $A$, there is a number $C>0$ such that

$$
|a b|_{B} \leq\left. C^{|a|}\right|_{A}|b|_{B}
$$

for all $a \in A, b \in B$ (see $[1]$, proof of Theorem 2.3). Analogously, if $B$ is a right ideal in $A$ and condition (1.2) holds, there is some $C>0$ such that 
$(1.5)$

$$
|b a|_{B} \leq C|b|_{B}|a|_{A}
$$

for all $a \in A, b \in B$ (see [1], proof of Theorem 2.3). This and the proof of [1], Proposition 2.1 give

Proposition (1.6). Let $A$ be semi-simple and $B$ be a dense subalgebra which is a Banach algebra under its own norm. Then B is a left [right] ideal in A if and only if (1.2) and (1.4) [(1.2) and (1.5)] hold. (For correctness suppose $a \in B$ in (1.4) and (1.5)).

\section{Ideals, Approximate units in ideals}

Definition. A Banach algebra A has approximate left (right) units, if $a \in \overline{A a}^{A}\left(a \in \overline{a A}^{A}\right)$ for every $a \in A$.

Let $B$ be an abstract Segal algebra in $A$.

(2.1) If I is a closed left (right) ideal of $A$, then $I \cap B$ is a closed left (right) ideal of B.

(2.2) If $J$ is a closed left (right) ideal of $B$, then $\bar{J}^{A}$ is a closed left (right) ideal of $A$.

This is straightforward (for one part use (1.2), for the other use density of $B$ in $A$ ).

Theorem (2.3). Let B be an abstract Segal algebra in A and let A and $B$ have approximate right units. Then the mapping $J \forall \rightarrow J^{A}$ is bijective from the set of all closed right (two-sided) ideals in B onto the set of all closed right (two-sided ideals in A. The inverse mapping is $|\mapsto| \cap B$.

Part of this Theorem was proved by J.T. Burnham in [2], Theorem 1.1. Using this result, H. Reiter established the Theorem for pseudosymmetric Segal algebras over a locally compact group in [4], $\S 9$, Theorem 1. His proof applies in fact to the general case. 
The statement for two-sided ideals follows from that for right ideals, since the mappings $J \mapsto J^{A}$ and $I \mapsto I \cap B$ carry closed two-sided ideals into closed two-sided ideals.

J. T. Burnham has given an example which shows that Theorem (2.3) may fail in the absence of approximate units. A sufficient condition for $A$ and $B$ to have approximate right units is for instance that $A$ has a bounded right approximate identity and B is a two-sided ideal in $A$ such that B.A is dense in B. Another sufficient condition obviously is that B has I I A-bounded approximate right units. Approximate units are not needed, if we consider maximal modular left ideals (see Theorem (3.7)).

Theorem (2.4). Let B be an abstract Segal algebra in $A$ and let $A$ and $B$ have approximate right units. Let I be a two-sided closed ideal in $A$ and $J=I \cap B$ be the corresponding two-sided closed ideal in $B$. Then I has approximate right units if and only if $J$ has approximate right units.

This Theorem has been proved by $H$. Reiter in the case of a Segal algebra over a locally compact abelian group. We essentially follow Reiter using a different condition to characterize the existence of approximate units and avoiding the assumption of commutativity by a small change in the proof ${ }^{+)}$. The Theorem settles the question raised by Reiter in $[4]$ : Theorem 1 in $[4], \& 16$ holds for general locally compact groups if we suppose the Segal algebra to be pseudosymmetric.

The proof of the Theorem is done in several steps.

If $D$ is a Banach algebra and $D^{\prime}$ is the sDace of all continuous I inear functionals on $D$, we define for $e \in D$ and $\varphi \in D^{\prime}$ linear functionals $e \varphi$ and $\varphi e \in D^{\prime}$ by

$$
\begin{aligned}
& \operatorname{e\varphi }(g)=\varphi(\text { eg }) \\
& \varphi e(g)=\varphi(g e),
\end{aligned}
$$

$g \in D . I t$ is immediately checked that $(e d) \varphi=d(e \varphi)$ and $(e \varphi) d=e(\varphi d)$ for $e, d \in D$ and $\varphi \in D^{\prime}$.

+) Meanwhile there is a short proof of this Theorem by Feichtinger. 
Lemma (2.5). If $B$ is an abstract Segal algebra in $A$ and if $b \in B$ and $\varphi \in B^{\prime}$, we have $\varphi b \in A^{\prime}$, by which we mean that $\varphi b$ is continuous with respect to the norm I IA and so can be extended to a continuous linear functional on $A$.

Proof: For $\varphi \in B^{\prime}$ and $b, c \in B$ we have

$$
\begin{aligned}
& |\varphi b(c)|=|\varphi(c b)| \leq|\varphi||c b|_{B} \leq C|\varphi||c|_{A}|b|_{B} \\
& \text { by (1.4). }
\end{aligned}
$$

Definition.

A subset $M$ of a Banach algebra $D$ satisfies condition $\left(r_{D}\right)\left[\left(I_{D}\right)\right]$, if for $m \in M$ and $\varphi \in D^{\prime}$ with $m \varphi \perp M[\varphi m \perp M]$ we have $\varphi(m)=0$.

Proposition (2.6). Let $M$ be a subalgebra of a Banach algebra D. Then $M$ satisfies ( $\left.r_{D}\right)\left[\left(I_{D}\right)\right]$ if and only if $M$ has approximate right units [approximate left units].

This is proved easily by means of the Hahn-Banach-Theorem.

Proposition (2.7). Let B be an abstract Segal algebra in A and let A have approximate right units. Let I be a closed two-sided ideal in $A$ and let $J=I \cap B$. Then, if $J$ satisfies condition $\left(r_{B}\right)$, I satisfies $\left(r_{A}\right)$.

Proof: Let $J$ satisfy $\left(r_{B}\right)$. Let $i \in I$ and $\varphi \in A^{\prime}$ be such that $i \varphi \perp 1$. Since $A$ has approximate right units and $B$ is dense in $A$, we can find a sequence $\left\{u_{n}\right\}$ in $B$ such that $I i u_{n}-i I_{A} \rightarrow 0$. We have $i u_{n} \in B \cap 1=J$, since $B$ is a left ideal and $I$ is a right ideal in $A$, and

$$
\left(i u_{n}\right) \varphi=u_{n}(i \varphi) \perp 1,
$$

since 1 is also a left ideal. So $\left(i u_{n}\right) \varphi \perp J$ since $J \subset 1$. Condition $\left(r_{B}\right)$ for $J$ implies $\varphi\left(i u_{n}\right)=0$, hence $\varphi(i)=0$. So I satisfies condition $\left(r_{A}\right)$.

Proposition (2.8). Let B be an abstract Segal algebra in A and let $B$ have approximate right units. Let $J$ be a closed right ideal in $B$ 
and let $1=J^{A}$. Then, if I satisfies $\left(r_{A}\right)$, J satisfies $\left(r_{B}\right)$.

Proof: Let I satisfy $\left(r_{A}\right)$. Let $j \in J$ and $\varphi \in B^{\prime}$ be such that $j \varphi \perp J$. Since $B$ has approximate right units, there is a sequence $\left\{u_{n}\right\}$ in $B$ such that $\mid j u_{n}-j I_{B} \rightarrow 0$; then

$$
j\left(\varphi u_{n}\right)=(j \varphi) u_{n} \perp J,
$$

since $J$ is a right ideal in B. According to Lemma (2.5) we have $\varphi u_{n} \in A^{\prime}$. So, by continuity we get $j\left(\varphi u_{n}\right) \perp j^{A}=1$. Because of $j \in J C I$ and condition $\left(r_{A}\right)$ for I we obtain $\varphi\left(j u_{n}\right)=\varphi u_{n}(j)=0$, hence $\varphi(j)=0$. So $J$ satisfies condition $\left(r_{B}\right)$.

The proof of Theorem (2.4) follows from Proposition (2.6), Proposition (2.7) and Proposition (2.8). The reader will have noticed that this proof does not work for right ideals since we used two-sidedness in the proof of Proposition (2.7).

\section{Strictly irreducible representations, Maximal modular left ideals}

B. A. Barnes has shown in [1] that two Banach *-algebras $A$ and $B$ have identical Hilbert space representation theory, if $\mathrm{B}$ is an abstract Segal algebra in A. The corresponding statement holds for strictly irreducible representations on linear vector spaces:

Proposition (3.1). Let B be an abstract Segal algebra in A. The restriction map $\pi \mapsto \pi_{B}$ establishes a bijective correspondence between the strictly irreducible representations of $A$ and those of $B$.

Proof: If $\pi$ is a strictly irreducible representation $\neq 0$ of $A$, then $\pi I_{B}$ is a strictly irreducible representation $\neq 0$ of $B$ by Lemma (1.3). If $\pi_{0}$ is a strictly irreducible representation $\neq 0$ of $B$ on a linear space $E$ and if $\xi \in E, \xi \neq 0$, we define $\pi$ by 


$$
\pi(a) \pi_{0}(b) \xi=\pi_{0}(a b) \xi
$$

for $a \in A, b \in B$. Then $\pi$ is well defined: the annihilator $N$ of $\xi$ in $B$ is a maximal modular left ideal in B, in particular it is closed in B. By (1.4) and density of B in A, N is a left ideal in $A$ which means that $\pi_{0}(b) \xi=0$ implies $\pi_{0}(a b) \xi=0$. So $\pi$ is well defined. By the way one easily checks that $\pi$ does not depend on the choice of $\xi$. It is immediate that the mappings

$$
\pi_{0} \mapsto \pi
$$

and

$$
\pi \mapsto \pi I_{B}
$$

are inverse to each other.

Corollary. $\quad \operatorname{Rad} B=(\operatorname{Rad} A) \cap B$.

Theorem (3.3). Let B be an abstract Segal algebra in A.

(i) If $A$ is semi-simple, so is $B$.

( $i$ i) If $B$ is semi-simple, then $A$ is semi-simple if and only if $A$ satisfies the condition

$$
\{a \in A \mid a A=\{0\}\}=\{0\} .
$$

Proof:

(i) follows from the Corollary above.

( $i$ i) Suppose that $B$ is semi-simple and $A$ satisfies (N). Let a $\in \operatorname{Rad} A$. Then $a B \subset B \frown \operatorname{Rad} A=\operatorname{Rad} B=\{0\}$. By density $a A=\{0\}$. So $a=0$, which means that $A$ is semi-simple. Conversely, if $A$ is semi-simple, then clearly condition ( $N$ ) holds since we always have

$$
\{a \in A \mid a A=\{0\}\} \subset \operatorname{Rad} A .
$$

By means of Proposition (3.1) we can establish a bijective correspondence for maximal modular left ideals. To each maximal modular left 
ideal $J$ of $B$ we assign a maximal modular left ideal $j$ of $A$ by the following method: Let $\pi_{0}$ be a strictly irreducible representation $\neq 0$ of $B$ on some linear space $E$ and let $\xi \in E, \xi \neq 0$, be a vector whose annihilator

$$
\left\{b \in B \mid \pi_{0}(b) \xi=0\right\}
$$

is equal to $J$ (for instance take $\pi_{0}$ to be the left regular representation of $\mathrm{B}$ on $\mathrm{B} / \mathrm{J}$ and take $\xi=u+J$ where $u$ is a right identity for $B$ modulo J). Consider the unique extension $\pi$ of $\pi_{0}$ to $A$ according to Proposition (3.1) and denote by $\tilde{J}$ the annihilator of $\xi$ in A. Clearly $\tilde{\jmath}$ is a maximal modular left ideal in $A$.

Proposition (3.4). Let B be an abstract Segal algebra in A. The map $J \mapsto \tilde{J}$ just defined is bijective from the set of all maximal modular left ideals of $B$ onto the set of all maximal modular left ideals of $A$.

Proof: Let $J$ be a maximal modular left ideal in B. We first show that $\tilde{J}$ does not depend on the choice of $\pi_{0}$ and $\xi$ in the above construction. Let $\pi_{0}$ and $\pi_{0}^{\prime}$ be strictly irreducible representations $\neq 0$ of $B$ and $l e t \xi$ and $\xi^{\prime}$ be vectors $\neq 0$ of the corresponding representation spaces $E$ and $E^{\prime}$, such that $J=\left\{b \in B \mid \pi_{0}(b) \xi=0\right\}=\left\{b \in B \mid \pi_{0}^{\prime}(b) \xi^{\prime}=0\right\}$

Then the map

$$
\begin{gathered}
\varphi: \quad E \longrightarrow E^{\prime} \\
\pi_{0}(b) \xi \mapsto \pi_{0}^{\prime}(b) \xi^{\prime}
\end{gathered}
$$

is a well defined linear isomorphism by which $\pi_{0}$ and $\pi_{0}^{\prime}$ are equivalent, as is easily checked. If $\pi$ and $\pi^{\prime}$ are the extensions of $\pi_{0}$ and $\pi_{0}^{1}$ to $A$, these representations are also equivalent by $\varphi$ because of (3.2). So we have

(3.5) $\{a \in A \mid \pi(a) \xi=0\}=\left\{a \in A \mid \pi^{\prime}(a) \varphi \xi=0\right\}$

However $\varphi \xi=\xi^{\prime}$ : let $e \in B$ be such that $\pi_{0}(e) \xi=\xi$.

Then we have for $a l l b \in B$ : 


$$
\begin{aligned}
\pi_{0}^{\prime}(b)\left(\pi_{0}^{\prime}(e) \xi^{\prime}-\xi^{\prime}\right) & =\pi_{0}^{\prime}(b e-b) \xi^{\prime} \\
& =\varphi \pi_{0}(b e-b) \xi \\
& =0
\end{aligned}
$$

So $\pi_{0}^{\prime}(e) \xi^{\prime}-\xi^{\prime}=0$ since $\pi_{0}^{\prime}$ is strictly irreducible $\neq 0$. Hence

$$
\varphi \xi=\varphi \pi_{0}(e) \xi=\pi_{0}^{\prime}(e) \xi^{\prime}=\xi^{\prime} .
$$

This and (3.5) show that $\tilde{j}$ does not depend on the choice of $\pi_{0}$ and $\xi$. From Proposition (3.1) it is clear that $\tilde{J} \cap B=J$. Now let 1 be a maximal modular left ideal of A. Applying Proposition (3.1) to the left regular representation of $A$ on $A / I$, we see that $I \cap B$ is a maximal modular left ideal of $B$ and that $\widetilde{I \cap B}=1$.

Proposition (3.6). We always have $\tilde{J}=J^{A}$.

Proof: If $J$ is a maximal modular left ideal in B, then clearly $J^{A} \subset \tilde{J}$. To prove the converse inclusion we consider $\pi_{0}$ to be the left regular representation of $B$ on $B / J$ and $\xi$ to be the class $u+J$ where $u$ is a right identity for B modulo $J$. Since $\pi_{0}(b) \xi=b+J$ for $b \in B$, the extended representation $\pi$ of $A$ on $B / J$ is given according to (3.2) by

$$
\pi(a)(b+J)=a b+J,
$$

$a \in A, b \in B$. Now let $a \in \tilde{J}=\{c \in A \mid c u \in J\}$. Let $\left\{b_{n}\right\}$ be a sequence in $B$ such that

$$
\left|b_{n}-a\right|_{A} \rightarrow 0 \text {. }
$$

We have $b_{n} u-b_{n} \in J$ which implies au - a $\in J^{A}$. since by assumption au $\in J$, we have $a \in J^{A}$.

Proposition (3.4) and Proposition (3.6) give

Theorem (3.7). Let B be an abstract Segal algebra in A. Then the map $J \mapsto J^{A}$ is bijective from the set of all maximal modular left ideals in B onto the set of all maximal modular left ideals in A. 
For A commutative this was proved in [2] by J. T. Burnham. In the general case, a partial result but which applies to all closed modular left ideals was obtained by B. A. Barnes in $[1]$

Theorem 3.1 .

\section{LITERATURE}

$$
\begin{aligned}
& \text { [1] B. A. Barnes, } \\
& {[2] \text { J.T. Burnham, }} \\
& \begin{array}{l}
{[3] \text { E. Hewitt and }} \\
\text { K. A. Ross }
\end{array} \\
& \text { [4] H. Reiter, } \\
& \text { [5] C. E. Rickart, } \\
& \text { Banach algebras which are ideals in a } \\
& \text { Banach algebra, } \\
& \text { Pac. J. Math. } 38 \text { (1971), 1-7. } \\
& \text { Closed ideals in subalgebras of Banach } \\
& \text { algebras I, } \\
& \text { Proc. Amer. Math. Soc. } 32 \text { (1972), 551-555. } \\
& \text { Abstract Harmonic Analysis 11, } \\
& \text { Springer-Verlag, } 1970 . \\
& \text { L'-Algebras and Segal Algebras, } \\
& \text { Lecture Notes in Mathematics } 231 \text {, } \\
& \text { Springer-Verlag, } 1971 . \\
& \text { Banach Algebras, } \\
& \text { D. Van Nostrand, } 1960 . \\
& \text { [6] J. Cigler, } \\
& \text { Normed ideals in } L^{1}(G) \text {, } \\
& \text { Indag. Math. } 31 \text { (1969), } 273-282 \\
& \text { [7] H. G. Feichtinger, }
\end{aligned}
$$

\footnotetext{
Michael Leinert

Fakultät für Mathematik Universität Bielefeld

$\frac{48 \text { Bielefeld }}{\text { Kurt-Schumacher-Str. } 6}$

W. -Germany
}

(Received May 3, 1973) 